\title{
We need those stinking badges!: Clinicians' competence as evidence based providers should be verified
}

\author{
Robert D. Friedberg, Ph.D., ABPP, ACT \\ Palo Alto University, Founding Editor for Contemporary Behavioral Health Care
}

In the classic 1948 US movie, Treasure of the Sierra Madre [1], Fred C Dobbs asks bandits pretending to be police officers to show him their badges. The outlaw leader indignantly replies, "Badges, we don't need no stinking badges!" This timeless exchange has been co-opted by many to emphasize that they do not have to comply with demands to verify their credentials. Perhaps, many community practitioners may adopt a similar stance and attitude. However, badges are important; they signify achievement and competence.

Unfortunately, self-branding is common in behavioral health care. Self-branding is essentially giving oneself a badge regardless of either training or competence. The lay public and other professionals are left to believe practitioners are genuinely credentialed in some evidence based approach. Problems in practitioners branding themselves as evidence based providers are well-documented [2-4]. Simply, there is little relationship between what many practitioners say they do in session and how they actually practice.

Patient care declines when self-branding flourishes. The effectiveness of psychotherapy like other health care services is dependent on delivery methods. For instance, if a batch of flu vaccine is administered at a low dose or incompetently injected into a patient's upper arm, its inoculating effects are diminished. Similarly, if CBT or any other form of evidence based psychotherapy is conducted incompetently or at an inappropriate intensity level, there is little reason to expect good results. The problem is not with the intervention, whether it is a vaccination or CBT, but with the individual service provision.

Authentic credentialing is the way forward to standardize delivery of care. There are multiple ways to obtain reputable badges. Board certification in Cognitive and Behavioral Psychotherapy is available from the American Board of Professional Psychology (www.abpp.org). Certification as a Diplomate in Cognitive Therapy can be obtained from the Academy of Cognitive Therapy (www.academyofct.org). Certificates of specialized training are available from the Beck Institute for Cognitive Behavioral Therapy (www.beckinstitute.org), the leading training center in the world, as well as many reputable national and international Centers for Cognitive Therapy. Most, if not all, of these credentialing bodies require multiple work samples to document actual clinical performance. There is some suggestion that in the future, practitioners will be subject to on-going periodic reviews of their work to substantiate claims of practicing evidence-based methods. Indeed, I believe these are welcome developments. We need those stinking badges!

Copyright: (C2015 Friedberg RD. This is an open-access article distributed under the terms of the Creative Commons Attribution License, which permits unrestricted use, distribution, and reproduction in any medium, provided the original author and source are credited.
The behavioral health care community needs to look beyond face value of practitioners' claims and pull back the curtains to inspect what is being done in clinicians' offices. Moreover, public education efforts should be directed toward informing potential patients about how to find a therapist who genuinely provides evidence-based care and what to expect from such services. In last few years, the behavioral health care community has done laudable work improving access to many vulnerable individuals. We now must redouble our efforts to ensure that clinical accountability accompanies this increased access to services.

This is my first of what I hope will be many Editorial Point of Views (POVs). As Founding Editor of Contemporary Behavioral Health Care, I am committed to offering readers quality scholarship that lives at the cutting-edge of research, theory, clinical practice, and policy. The journal is especially interested in empirical research, theoretical articles, critical literature reviews, clinical practice pieces, and policy issues in behavioral health care. Accordingly, each manuscript should clearly contain implications for contemporary behavioral health care. Please join me in this important work by submitting your own original work pertaining to Contemporary Behavioral Health Care. Submission requirements are found on our website. I look forward to receiving your submissions and any commentary on the Editorial POVs.

\section{References}

1. Huston J (1948) The Treasure of the Sierra Madre [Motion Picture]. United States: Henry Blake, Jack Warner [Producers]

2. Creed TA, Wolk CB, Feinberg B, Evan AC, Beck AT (2014) Relationship between community therapists self-report of a cognitive behavioral therapy orientation and observed skills. Administration and Policy in Mental Health 41: 1-8.

3. Friedberg RD (2014) Training matters: The importance of delivering authentic CBT with youth. J Psychology and Clinical Psych 1: 00047.

4. McKay D (2014) So you say you are an expert?: False CBT identity harms our hardearned gains. The Behavior Therapist 37: 215-216.
Correspondence to: Robert D. Friedberg, Ph.D., ABPP, ACT (Founding Fellow), Professor, Director, PAU Center for the Study and Treatment of Anxious Youth http://www.paloaltou.edu/CSTAY, Interim Head, Child and Family Emphasis Area , Pacific Graduate School of Psychology/Palo Alto University, 1791 Arastradero Rd. Palo Alto, CA. 94304, Tel: 650-961-9300 Ext. 3617, E-mail: rfriedberg@paloaltou.edu

Received: April 03, 2015; Accepted: April 05, 2015; Published: April 08, 2015 
Friedberg RD (2015) We need those stinking badges!: Clinicians' competence as evidence based providers should be verified 